\section{Repercusión de la anemia preoperatoria en pacientes a quienes se realiza nefrectomía simple retroperitoneal}

Carrillo-Córdova LD, ${ }^{1}$ Lemus-Mena GR, ${ }^{1}$ Rodríguez-Robles $\mathrm{J},{ }^{1}$ JiménezVillavicencio JM, ${ }^{1}$ Vitar-Sandoval J, ${ }^{1}$ Sarabia-Estrada RC, ${ }^{1}$ Carrillo-Córdova $\mathrm{JR},{ }^{2}$ Hernández-Farías MA, ${ }^{1}$ Rosas-Ramírez $\mathrm{A},{ }^{1}$ Virgen-Gutiérrez JF, ${ }^{1}$ JaspersenGastelum J, ${ }^{1}$ Garduño-Arteaga $\mathrm{ML}^{2}$

Resumen

ANTECEDENTES: la incidencia de anemia en pacientes quirúrgicos es alta y estrechamente relacionada con la enfermedad de base (2095\%). En los pacientes oncológicos puede llegar a 95\% y en el absceso renal alcanza cifras de $90 \%$, con repercusión directa en la morbilidad y mortalidad posoperatorias.

OBJETIVO: describir los resultados de las nefrectomías simples retroperitoneales abiertas, secundarias a litiasis, y comparar si los pacientes con anemia previa a la nefrectomía tienen mayor tiempo quirúrgico, sangrado transoperatorio, complicaciones, necesidad de transfusión y de ingreso a la unidad de terapia intensiva, días de estancia hospitalaria, infección de herida quirúrgica que los pacientes sin anemia.

MATERIALES Y MÉTODOS: estudio retrospectivo, descriptivo y comparativo de pacientes con anemia preoperatoria versus con cifras de hemoglobina normal. Análisis de las variables transoperatorias (tiempo quirúrgico, sangrado transoperatorio) y posquirúrgicas (necesidad de terapia intensiva y de transfusión, infección de herida quirúrgica y días de estancia hospitalaria).

RESULTADOS: se estudiaron 58 pacientes (24 masculinos y 34 femeninos) con antecedente de nefrectomía simple abierta y media de edad de 46 años. La media de sangrado transoperatorio fue de 604.9 $\mathrm{mL}$ y la media de tiempo quirúrgico de $158.6 \mathrm{~min}$. Hubo diferencias estadísticamente significativas para las variables de necesidad de transfusión $(p=0.0016)$ y de cuidados en la unidad de terapia intensiva $(p=0.0092)$, sin diferencias estadísticamente significativas para el sangrado transoperatorio, tiempo quirúrgico, días de estancia hospitalaria, lesión a algún órgano de manera transoperatoria o infección de herida quirúrgica.

CONCLUSIONES: los pacientes con anemia preoperatoria y nefrectomía simple retroperitoneal tienen mayor necesidad de transfusión perioperatoria y atención en una unidad de cuidados intensivos que quienes tienen cifras de hemoglobina normales. En estos pacientes es indispensable el estudio de la anemia y su reversión mediante suplementos con hierro o eritropoyetina previa al procedimiento quirúrgico para mejorar su pronóstico, y así prevenir la necesidad de transfusión perioperatoria, que se asocia con mayor morbilidad y mortalidad.

PALABRAS CLAVE: nefrectomía, anemia, complicaciones, exclusión renal, litiasis.

\begin{abstract}
${ }^{1}$ Departamento de Urología, Hospital General de México Dr. Eduardo Liceaga, Ciudad de México, México. ${ }^{2}$ Departamento de Cirugía Plástica y Reconstructiva, Hospital General Dr. Manuel Gea González, Ciudad de México, México.
\end{abstract}

Recibido: diciembre 2016

Aceptado: enero 2017

Correspondencia

Dr. Luis Daniel Carrillo Córdova

carrillocor@gmail.com

Este artículo debe citarse como Carrillo-Córdova LD, Lemus-Mena GR, RodríguezRobles J, Jiménez-Villavicencio JM, Vitar-Sandoval J, Sarabia-Estrada RC, et al. Repercusión de la anemia preoperatoria en pacientes a quienes se realiza nefrectomía simple retroperitoneal. Rev Mex Urol. 2017 mar;77(2):89-96. 


\section{Impact of preoperative anemia on patients that underwent retroperitoneal simple nephrectomy}

Carrillo-Córdova LD, ${ }^{1}$ Lemus-Mena GR, ${ }^{1}$ Rodríguez-Robles $\mathrm{J},{ }^{1}$ JiménezVillavicencio JM ${ }^{1}$ Vitar-Sandoval J, ${ }^{1}$ Sarabia-Estrada RC, ${ }^{1}$ Carrillo-Córdova $\mathrm{JR},{ }^{2}$ Hernández-Farías MA, ${ }^{1}$ Rosas-Ramírez $\mathrm{A},{ }^{1}$ Virgen-Gutiérrez JF, ${ }^{1}$ JaspersenGastelum J, ${ }^{1}$ Garduño-Arteaga $\mathrm{ML}^{2}$

\begin{abstract}
BACKGROUND: the incidence of anemia in surgical patients is high and is closely related to the underlying disease (20-95\%). In oncologic patients, it can reach $95 \%$ and in cases of renal abscess it reaches figures of $90 \%$. It has a direct impact on postoperative morbidity and mortality.
\end{abstract}

OBJECTIVE: to describe the results of open retroperitoneal simple nephrectomy secondary to lithiasis and to determine if patients with anemia prior to nephrectomy have longer surgery duration, greater intraoperative bleeding, more complications, a greater need for transfusion, higher admission to the intensive care unit, longer hospital stay, and increased surgical wound infection, compared with patients that do not present with anemia.

MATERIALS AND METHODS: a retrospective, descriptive, and comparative study was conducted on patients with preoperative anemia versus those with normal hemoglobin. Intraoperative variables (surgery duration, intraoperative blood loss) and postoperative variables (the need for intensive care and transfusion, surgical wound infection, and days of hospital stay) were analyzed.

RESULTS: fifty-eight patients that underwent open simple nephrectomy were studied (24 men and 34 women). The mean age of the patients was 46 years, mean intraoperative blood loss was $604.9 \mathrm{~mL}$, and mean surgery duration was $158.6 \mathrm{~min}$. There were statistically significant differences in relation to the variables of need for transfusion $(p=$ $0.0016)$ and admission to the intensive care unit $(\mathrm{p}=0.0092)$. There were no statistically significant differences regarding intraoperative blood loss, surgery duration, days of hospital stay, intraoperative injury to an organ, or surgical wound infection.

CONCLUSIONS: The patients with preoperative anemia that underwent retroperitoneal simple nephrectomy had a greater need for perioperative transfusion and critical care in the intensive care unit, compared with the patients with normal hemoglobin values. The study and correction of anemia in those patients through iron supplementation or the use of erythropoietin prior to the surgical procedure is indispensable to improve outcome and prevent the need for perioperative transfusion, which is associated with greater morbidity and mortality.

KEY WORDS: nephrectomy, anemia, complications, non-functioning kidney, lithiasis

\footnotetext{
1 Departamento de Urología, Hospital General de México Dr. Eduardo Liceaga, Ciudad de México, México.

2 Departamento de Cirugía Plástica y Reconstructiva, Hospital General Dr. Manue Gea González, Ciudad de México, México.
}

Correspondence

Dr. Luis Daniel Carrillo Córdova carrillocor@gmail.com 


\section{ANTECEDENTES}

La anemia, según la OMS, se define como valores de hemoglobina menores a $13 \mathrm{mg} / \mathrm{dL}$ en hombres y menos de $12 \mathrm{mg} / \mathrm{dL}$ en mujeres. Su prevalencia es de 17 a $63 \%$ en pacientes de edad avanzada en quienes altera la eficacia del aporte tisular de oxígeno. La anemia es un síntoma o comorbilidad asociada con múltiples padecimientos. En pacientes mayores a 65 años, un tercio es de origen ferropénico, un tercio es por anemia originada por procesos inflamatorios crónicos y el tercio restante es de origen desconocido. ${ }^{1}$

En los pacientes quirúrgicos la incidencia de anemia es alta, estrechamente relacionada con la enfermedad de base (20-95\%). En los pacientes oncológicos puede ser tan alta como $95 \%,{ }^{2}$ y en las enfermedades urológicas lo más estudiado es el absceso renal, con incidencia de anemia de $90 \%{ }^{3}$ y repercusión directa en la morbilidad y mortalidad posoperatorias.

Según el estudio RECIDON (estudio epidemiológico español de la anemia preoperatoria en pacientes con cirugía oncológica) la anemia está debidamente documentada como factor predictivo de riesgo de transfusión. ${ }^{4}$ La fisiopatología de la anemia, en enfermedad crónica, implica una interacción intensa entre el tumor o proceso crónico y el sistema inmunológico; esta interacción conduce a la activación del macrófago y al incremento en la expresión de varias citocinas y hepcidina. En trastornos asociados con esa alteración se han reportado concentraciones elevadas de citocinas y hepcidina que se consideran factores potencialmente implicados en la fisiopatología.

El incremento de citocinas inflamatorias, como: interferón gamma (INF g), interleucina 1 (IL-1) y factor de necrosis tumoral (FNT) tiene una repercusión negativa en la diferen- ciación de los precursores eritroides, en la producción de eritropoyetina y contribuye al defecto en la utilización del hierro y deficiente absorción intestinal de éste. ${ }^{5}$ En el caso de los padecimientos urológicos, como: litiasis e infecciones con exclusión renal secundaria, el déficit de producción de eritropoyetina, junto con el aumento de pérdidas sanguíneas insensibles, contribuye al inicio de la anemia prequirúrgica.

La respuesta inflamatoria sistémica, desencadenada por la propia agresión quirúrgica, independiente de la hemorragia perioperatoria, ocasiona que durante el periodo posoperatorio sea frecuente la hiposideremia y la disminución de los valores de transferrina e IST, con incremento de la ferritina. Esto implica un marcado descenso de la disponibilidad y absorción del hierro en las primeras 2 a 6 semanas de posoperatorio, lo que dificulta la eritropoyesis. Este mecanismo, sumado al déficit nutricional de los pacientes con enfermedades crónicas, en general no afecta solo a la eritropoyesis sino a varios aspectos del sistema inmunitario. ${ }^{6}$ Por la frecuencia con que se manifiesta, y por la importante repercusión clínica de la anemia, se torna en un complejo procedimiento de atención del paciente quirúrgico; por esto muchas veces se recurre a la transfusión sanguínea, como una forma rápida y eficaz de restaurar la hemostasia. ${ }^{7}$

El objetivo de esta investigación es: describir los resultados de las nefrectomías simples retroperitoneales abiertas, secundarias a litiasis en el Hospital General de México Dr. Eduardo Liceaga y determinar si los pacientes con anemia previa a la nefrectomía tuvieron mayor tiempo quirúrgico, sangrado transoperatorio, complicaciones (según la clasificación de Clavien-Dindo ), ${ }^{8}$ necesidad de transfusión, días de estancia hospitalaria, de terapia intensiva e infección de herida quirúrgica que los pacientes sin anemia. 


\section{MATERIALES Y MÉTODOS}

Estudio retrospectivo, descriptivo y comparativo de pacientes con anemia preoperatoria versus con cifras de hemoglobina normal atendidos en el Hospital General de México Dr. Eduardo Liceaga entre los meses de enero de 2010 y mayo de 2016. Se buscaron expedientes en el archivo clínico que coincidieran con el código ICD-9 para nefrectomía. En todos los pacientes el acceso quirúrgico fue retroperitoneal, por lumbotomía; a todos se les administró antibiótico al momento de la cirugía.

Para fines de estudio los pacientes se dividieron en dos grupos: grupo 1, pacientes con anemia previa (según criterios de la OMS) a la nefrectomía retroperitoneal; grupo 2, pacientes con nefrectomía secundaria a exclusión renal con litiasis, con cifras normales de hemoglobina.

Variables de estudio: edad, sexo, peso, localización del lito. Variables transoperatorias: tiempo quirúrgico y sangrado transoperatorio. Variables posquirúrgicas: necesidad de terapia intensiva y de transfusión, infección de herida quirúrgica y días de estancia hospitalaria. Los pacientes del grupo 1 se compararon con los del grupo 2. Para las variables paramétricas se utilizó t de Student y $\chi^{2}$, con corrección de Yates, para los datos no paramétricos.

\section{RESULTADOS}

Se evaluaron 110 expedientes de pacientes con nefrectomías abiertas de los que se excluyeron 52 por carecer de diagnóstico de litiasis. Al final se incluyeron 58 pacientes con diagnóstico de exclusión renal confirmado mediante gammagrafía renal y litiasis, con media de edad de 46 años $(D E \pm 14.1)$ a quienes se realizó nefrectomía simple por un grupo de cirujanos expertos.
La localización del lito fue piélica en 32\%, ureteral $6.9 \%$ y coral $55.2 \%$. Variables transoperatorias: media de sangrado $604.9 \mathrm{~mL}$ $(\mathrm{DE} \pm 720 ; 20-5000 \mathrm{~mL}$ ) y media de tiempo quirúrgico $158.6 \min (\mathrm{DE} \pm 62 ; 55-318)$. Variables posoperatorias: 19 pacientes requirieron transfusión (32.8\%)(Clavien-Dindo II), 10 tuvieron (17.2\%) lesión vascular o a órgano sólido durante la cirugía (Clavien-Dindo III b), 13 ingresaron (22.4\%) a la unidad de terapia intensiva (Clavien-Dindo IV); media de estancia hospitalaria 7 días (DE $\pm 2.3: 2-10) ; 31$ (46.6\%) ( Clavien-Dindo II) con infección de la herida quirúrgica, un fallecimiento (1.7\%) (ClavienDindo V). Cuadro 1

Cuadro 1. Estadística descriptiva para 58 pacientes posoperados de nefrectomía simple retroperitoneal secundaria a litiasis

\begin{tabular}{|c|c|c|c|}
\hline Variable & $\mathbf{n}$ & Media & $(\%)$ \\
\hline $\begin{array}{l}\text { Sexo } \\
\text { Masculino } \\
\text { Femenino }\end{array}$ & $\begin{array}{l}24 \\
34\end{array}$ & $\begin{array}{l}\text { N/A } \\
\text { N/A }\end{array}$ & $\begin{array}{l}41.4 \% \\
58.6 \%\end{array}$ \\
\hline $\begin{array}{l}\text { Localización del lito } \\
\text { Piélica } \\
\text { Ureteral } \\
\text { Coral }\end{array}$ & $\begin{array}{c}19 \\
4 \\
32\end{array}$ & & $\begin{array}{c}32.8 \% \\
6.9 \% \\
55.2 \%\end{array}$ \\
\hline STO & 58 & $\begin{array}{c}609.9 \mathrm{~mL} \\
(\mathrm{DE} \pm 720, \text { rango } \\
20-5000)\end{array}$ & $\mathrm{N} / \mathrm{A}$ \\
\hline Tiempo quirúrgico & 58 & $\begin{array}{c}158.6 \min (\mathrm{DE} 62, \\
\text { Rango } 55-318)\end{array}$ & $\mathrm{N} / \mathrm{A}$ \\
\hline Transfusión & 19 & $N / A$ & $32.8 \%$ \\
\hline Lesión a órgano & 10 & $\mathrm{~N} / \mathrm{A}$ & $17.2 \%$ \\
\hline Necesidad de UTI & 13 & $\mathrm{~N} / \mathrm{A}$ & $22.4 \%$ \\
\hline EIH & 58 & $\begin{array}{l}7 \text { días (DE 2.3, } \\
\text { Rango 2-10) }\end{array}$ & $\mathrm{N} / \mathrm{A}$ \\
\hline Infección herida & 31 & N/A & $46.6 \%$ \\
\hline Muerte & 1 & $\mathrm{~N} / \mathrm{A}$ & $1.7 \%$ \\
\hline Anemia & 35 & $\mathrm{~N} / \mathrm{A}$ & $60.3 \%$ \\
\hline
\end{tabular}

STO: sangrado transoperatorio, UTI: unidad de cuidados intensivos, EIH: estancia intra hospitalaria 
Se encontraron 35 pacientes $(60.3 \%)$ con anemia preoperatoria, con media de hemoglobina de 10.2 ( \pm DE 2.5$)$ y $23(39.6 \%)$ con cifras de hemoglobina normales, con una media de 16 ( \pm DE 4.2). Un paciente del grupo 1 falleció (Clavien-Dindo V) durante el posoperatorio inmediato. En la comparación de estos dos se encontró una media de sangrado transoperatorio, en el grupo 1, de $748 \mathrm{~mL}(\mathrm{DE} \pm 868.9$ : 20-5000 mL), y en el grupo 2 de $386 \mathrm{~mL}(\mathrm{DE}$ \pm 307: 50-1600 mL). Para tiempo quirúrgico en el grupo 1 la media fue de $150.8 \mathrm{~min}$ (DE $\pm 65.4: 60-270$ ) y para el grupo $2: 166.2 \mathrm{~min}$ (DE $\pm 64.4: 60-318)$. Por lo que se refiere a los días de estancia intrahospitalaria la media del grupo 1 fue de 7.28 ( $D E \pm 3.5: 4-15)$, y para el grupo 2 de 5.9 días $(\mathrm{DE} \pm 2.23$ : 4-15). Para necesidad de transfusión en el grupo 1, 17 pacientes $(48.6 \%)$ la requirieron y en el grupo 2 , dos pacientes (8.7\%). Del grupo 1: 12 pacientes requirieron ingresar a la unidad de cuidados intensivos (34.3\%), y en el grupo 2, un paciente $(4.3 \%)$. En el grupo 1 en cuatro pacientes se documentó lesión vascular o a órgano (11.4\%) y en el grupo 2, en dos pacientes también se documentó $(8.7 \%) ; 22$ pacientes tuvieron infección de herida quirúrgica en el grupo 1 $(62.9 \%)$ y 9 pacientes $(39.1 \%)$ en el grupo 2 .

Cuadro 2. Comparación de las variables paramétricas en los dos grupos

$\begin{array}{lccc}\text { Variable } & & \text { Valor de } \mathbf{p} \\ & \text { Media } & \\ & \text { Grupo 1 } & \text { Grupo 2 } & \\ \text { STO } & 748 \mathrm{~mL} & 386 \mathrm{~mL} & 0.0602 \\ & (\mathrm{DE} \pm 868.9) & (\mathrm{DE} \pm 307) & \\ \text { Tiempo } & 150.8 \mathrm{~min} & 166.2 \mathrm{~min} & 0.3572 \\ \text { Qx } & (\mathrm{DE} \pm 65.4) & (\mathrm{DE} \pm 64.4) & \\ \text { EIH } & 7.28 & 5.9 \text { días } & 0.0990 \\ & (D E \pm 3.5) & (\mathrm{DE} \pm 2.23) & \\ & & \end{array}$

STO: sangrado transoperatorio, tiempo Qx.: tiempo quirúrgico de la incisión en piel al cierre de la herida, ElH: días de estancia hospitalaria, * diferencia estadísticamente significativa.
Cuadro 3. Comparación de las variables no paramétricas en los dos grupos

\begin{tabular}{|lccc|}
\hline Variable & & & Valor de $\mathbf{p}$ \\
\hline & $(n) \mathrm{y}(\%)$ & & \\
\hline Transfusión & Grupo 1 & Grupo 2 & \\
\hline UTI & $17(48.6 \%)$ & $2(8.7 \%)$ & $\mathbf{0 . 0 0 1 6 *}$ \\
\hline LOVTO & $4(11.4 \%)$ & $2(8.7 \%)$ & 1.000 \\
\hline IHQ & $22(62.9 \%)$ & $9(39.1 \%)$ & 0.1077 \\
\hline
\end{tabular}

UTI: necesidad de cuidado en unidad de cuidados intensivos, LOVTO: Lesión a órgano o lesión vascular en el transoperatorio, IHQ: infección de herida quirúrgica, * diferencia estadísticamente significativa.

Cuadros 2 y 3

Al hacer la comparación entre los dos grupos se encontraron diferencias estadísticamente significativas para las variables de necesidad de transfusión ( $p=0.0016)$ y de cuidados en la unidad de terapia intensiva ( $p=0.0092)$, no así para el sangrado transoperatorio, tiempo quirúrgico, días de estancia hospitalaria, lesión a algún órgano de manera transoperatoria o infección de herida quirúrgica.

\section{DISCUSIÓN}

Un paciente con anemia preoperatoria falleció, pero ninguno con hemoglobina normal. La repercusión de la anemia preoperatoria en la mortalidad perioperatoria puede ilustrarse con los pacientes testigos de Jehová, que no aceptan transfusiones por motivos religiosos. Un estudio retrospectivo, efectuado en 1958 con testigos de Jehová, llevados a cirugía no cardiaca, con concentración de hemoglobina menor o igual a 10, se asoció con mayor mortalidad perioperatoria. ${ }^{9}$

Los pacientes con anemia transoperatoria tienen mayor necesidad de transfusión que los de cifras de hemoglobina normales $\left(0.0016^{*}\right)$. En 
pacientes que aceptan la transfusión se ha reportado que la anemia preoperatoria es un factor predictor, significativo de transfusión, como se comunicó en este estudio. ${ }^{10,12}$

En la especialidad de Urología, la anemia ha sido extensamente estudiada, sobre todo en pacientes oncológicos. La coexistencia de anemia en pacientes a quienes se efectúa nefroureterectomía radical, cistectomía radical o resección de tumor vesical secundario a cáncer vesical no músculo invasor, se asocia con mayor riesgo de recurrencia oncológica, mayor mortalidad y menor supervivencia. No existen estudios que pongan de manifiesto la trascendencia de la anemia en pacientes con nefrectomías simples, secundarias a exclusión renal. ${ }^{13-15}$

La anemia preoperatoria y la necesidad de transfusión se asocian con morbilidad y mortalidad mayor en los pacientes posoperados, incluida la necesidad de atención especial en una unidad de terapia intensiva. En un análisis retrospectivo de 300,000 pacientes mayores de cirugía no cardiovascular, el hematocrito prequirúrgico $\leq 39 \%$ se asoció, de manera estadísticamente significativa, con mayor mortalidad. ${ }^{12}$ Estos hallazgos se confirmaron en un estudio subsecuente que, retrospectivamente, analizó 8000 pacientes de cirugía no cardiovascular que reportó que $40 \%$ de los que tuvieron anemia preoperatoria también informaron mayor índice de mortalidad. ${ }^{16}$

Con base en lo reportado en la bibliografía, y lo encontrado en nuestro estudio, se recomienda que los pacientes a quienes se efectuará nefrectomía tengan hemoglobina en los límites normales establecidos por la OMS (mujeres $\geq 12 \mathrm{~g} / \mathrm{dL}$, hombres $\geq 13 \mathrm{~g} / \mathrm{dL}$ ).

La anemia, sin una causa evidente, debe estudiarse porque puede coexistir algún otro padecimiento no diagnosticado. ${ }^{17,18}$ La biometría hemática y la química sanguínea deben analizarse minuciosamente en busca de deficiencias nutricionales, insuficiencia renal crónica o una enfermedad crónica responsable de enfermedad. Todos nuestros pacientes tenían litiasis, con exclusión renal, proceso que puede catalogarse como enfermedad crónica. Aunado a esto 34.5\% de nuestros pacientes tenían antecedente de absceso renal, padecimiento que se ha asociado con anemia en estudios previos, en los que se han reportado tasas tan altas como $90 \%{ }^{3}$

Al identificar un paciente con anemia, el primer paso es analizar el perfil de hierro y, en caso de detectar una anemia ferropénica, hay que estudiar si es secundaria a una deficiencia de hierro, a secuestro de hierro por un proceso inflamatorio crónico (como sucedió en la mayoría de nuestros pacientes), o una deficiencia funcional del hierro por alteraciones en la eritropoyetina. ${ }^{19}$

Cuando se detecta una deficiencia absoluta de hierro, el paciente debe enviarse al servicio de Gastroenterología en busca de sangrado del tubo digestivo, como causa de la pérdida de sangre. Si los estudios de laboratorio descartan la deficiencia de hierro, hay que solicitar la cuantificación de la creatinina sérica y tasa de filtración glomerular y si se demuestra insuficiencia renal crónica entonces se solicitará el apoyo del nefrólogo. ${ }^{20}$

Las deficiencias nutricionales tienen que tratarse en el estudio preoperatorio del paciente, e indicar hierro complementario cuando se demuestre que padece anemia secundaria a la deficiencia de hierro. La prescripción de hierro oral a pacientes con anemia preoperatoria ha demostrado disminuir la morbilidad y mortalidad en quienes serán operados. ${ }^{21-24}$ Un grupo de expertos recomendó, recientemente, administrar un suplemento con hierro, por vía oral o intravenosa, a todos los pacientes con anemia preoperatoria, considerando el tiempo disponible previo a la cirugía. ${ }^{25}$ 
La eritropoyetina está indicada en pacientes con anemia por deficiencia de hierro y en quienes se ha descartado una causa nutricional de la anemia. Son múltiples los ensayos clínicos con asignación al azar que demuestran las ventajas de la indicación de eritropoyetina a pacientes que de manera electiva serán intervenidos quirúrgicamente..$^{26-32}$

\section{CONCLUSIONES}

Los pacientes con anemia previa a la nefrectomía simple retroperitoneal tienen mayor necesidad de transfusión perioperatoria y atención en una unidad de cuidados intensivos que quienes tienen valores normales de hemoglobina. Para mejorar el pronóstico y prevenir la necesidad de transfusión perioperatoria, en estos pacientes es indispensable el estudio de la anemia y su correlación por medio de la complementación con hierro o eritropoyetina previa al procedimiento quirúrgico, y disminuir así la asociación de mayor morbilidad y mortalidad.

\section{Nota}

Los autores de este trabajo no tienen conflicto de interés alguno que declarar.

\section{REFERENCIAS}

1. Gaskell H, Derry S, Andrew Moore R, McQuay HJ, Prevalence of anaemia in older persons: systematic review. BMC Geriatr. 2008; 8:1

2. Warschkow R., Güller U., Köberle D., Müller S.A., Steffen T., Thurnheer M., Schmied B.M., Tarantino I. Perioperative blood transfusions do not impact overall and disease-free survival after curative rectal cancer resection: A propensity score analysis. Ann Surg. 2014; 259(1):131-8.

3. Maldonado-Alcaraz, E., Ixquiac-Pineda, G., López-Sámano, V., \& Serrano-Brambila, E. Absceso perirrenal: factores asociados a su desarrollo y mortalidad. Arch Esp Urol. 2008; 61(1), 07-12.

4. Durán, L., Moral, V., Basora, M., Colomina, M. J., Llau, J. V., Sánchez, C. A. \& Vila, M. Estudio epidemiológico de la anemia preoperatoria en pacientes sometidos a cirugía oncológica en España. Estudio RECIRON. Cir Esp. 2009; 85(1), 45-52.

5. Erslev A. Anemia of chronic disease. En: Beutler E, Lichtman MA, Cooller BS, Kipps TJ, eds. Williams Hematology. 5th ed. New York: Mc Graw- Hill; 1995.p. 518-24

6. Compés, M. C., Peris, P. G., Lesmes, I. B., Álvarez, M. C., \& Aparicio, P. B. Cáncer DE Estómago. Valoración Y Apoyo Nutricional Perioperatorio. APOYO NUTRICIONAL, 109. En http://www.chospab.es/libros/nutricion/libro_apoyo_nutri_fco_botella.pdf. Consultado: octubre 2016.

7. Muñoz M, Leal Noval SR, Garcia- Erce JA, Naveira E. Prevalencia y tratamiento de la anemia en el paciente crítico. Med Intensiva 2007; 31:388-9

8. Dindo D, Demartines N, Clavien PA. Classification of surgical complications: a new proposal with evaluation in a cohort of 6336 patients and results of a survey. Ann Surg. 2004; 240: 205-13.

9. Carson JL, Noveck H, Berlin JA, Gould SA. Mortality and morbidity in patients with very low postoperative $\mathrm{Hb}$ levels who decline blood transfusion. Transfusion. 2002; 42:812-8.

10. Khanna MP, Hebert PC, Fergusson DA. Review of the clinical practice literature on patient characteristics associated with perioperative allogeneic red blood cell transfusion. Transfus Med Rev. 2003; 17:110-9.

11. Myers $E, O$ 'Grady P, Dolan AM. The influence of preclinical anaemia on outcome following total hip replacement. Arch Orthop Trauma Surg. 2004 Dec; 124(10): 699-701.

12. Wu WC, Schifftner TL, Henderson WG, et al. Preoperative hematocrit levels and postoperative outcomes in older patients undergoing noncardiac surgery. J Am Med Assoc. $2007 ; 297: 2481-8$.

13. Xia L, Guzzo TJ. Preoperative Anemia and Low Hemoglobin Level Are Associated With Worse Clinical Outcomes in Patients With Bladder Cancer Undergoing Radical Cystectomy: A Meta-Analysis Clin Genitourin Cancer. 2016 Aug 29. pii: S1558-7673(16)30251-8. doi: 10.1016/j. clgc.2016.08.017.

14. Yeh HC, Chien TM, Wu WJ, et. Al. Is preoperative anemia a risk factor for upper tract urothelial carcinoma following radical nephroureterectomy Urol Oncol. 2016; 34(8):337.

15. Soria F, Moschini M, Abufaraj M, et. al. Preoperative anemia is associated with disease recurrence and progression in patients with non-muscle-invasive bladder cancer. Urol Oncol. 2016; 28. pii: S1078-1439(16)30362-3.

16. Beattie WS, Karkouti K, Wijeysundera DN, Tait G. Risk associated with preoperative anemia in noncardiac surgery: a single-center cohort study. Anesthesiology. 2009; 110:574-81.

17. Guralnik JM, Eisenstaedt RS, Ferrucci L, et al. Prevalence of anemia in persons 65 years and older in the United States: evidence for a high rate of unexplained anemia. Blood. 2004; 104:2263-8. 
18. Weiss G, Goodnough LT. Anemia of chronic disease. N Engl J Med.2005; 352:1011-23.

19. Mercuriali F, Zanella A, Barosi G, et al. Use of erythropoietin to increase the volume of autologous blood donated by orthopedic patients. Transfusion.1993; 33:55-60

20. Guyatt GH, Cook DJ, Jaeschke R, et al. Grades of recommendation for antithrombotic agents: American College of Chest Physicians Evidence-Based Clinical Practice Guidelines (8th Edition) Chest. 2008; 133:123-31S.

21. Andrews CM, Lane DW, BradLey JG. Iron pre-load for major joint replacement. Transfus Med. 1997; 7:281-6.

22. Cuenca J, Garcia-Erce JA, Martinez F, et al. Preoperative haematinics and transfusion protocol reduce the need for transfusion after total knee replacement. Int J Surg. 2007; 5:89-94.

23. Lidder PG, Sanders G, Whitehead E, et al. Pre-operative oral iron supplementation reduces blood transfusion in colorectal surgery-a prospective, randomised, controlled trial. Ann R Coll Surg Engl. 2007;89:418-21.

24. Okuyama M, Ikeda K, Shibata T, et al. Preoperative iron supplementation and intraoperative transfusion during colorectal cancer surgery. Surg Today.2005;35:36-40.

25. Beris P, Munoz M, Garcia-Erce JA, et al. Perioperative anaemia management: consensus statement on the role of intravenous iron. Br J Anaesth.2008; 100:599-604.

26. Besarab A, Bolton WK, Browne JK, et al. The effects of normal as compared with low hematocrit values in patients with cardiac disease who are receiving hemodialysis and epoetin. N Engl J Med. 1998; 339:584-90.

27. Drueke TB, Locatelli F, Clyne N, et al. Normalization of hemoglobin level in patients with chronic kidney disease and anemia. N Engl J Med.2006; 355:2071-84.

28. 5Singh AK, Szczech L, Tang KL, et al. Correction of anemia with epoetin alfa in chronic kidney disease. $\mathrm{N}$ Engl J Med. 2006; 355:2085-98.

29. Pfeffer MA, Burdmann EA, Chen CY, et al. A trial of darbepoetin alfa in type 2 diabetes and chronic kidney disease. N Engl J Med. 2009;361:2019-32.

30. Palmer SC, Navaneethan SD, Craig JC, et al. Metaanalysis: erythropoiesis-stimulating agents in patients with chronic kidney disease. Ann Intern Med.2010;153:23-33.

31. KDOQI Clinical Practice Guideline and Clinical Practice Recommendations for anemia in chronic kidney disease: 2007 update of hemoglobin target. Am J Kidney Dis. 2007;50:471-530.

32. Locatelli F, Nissenson AR, Barrett BJ, et al. Clinical practice guidelines for anemia in chronic kidney disease: problems and solutions. A position statement from Kidney Disease: Improving Global Outcomes (KDIGO) Kidney Int.2008;74:1237-40.

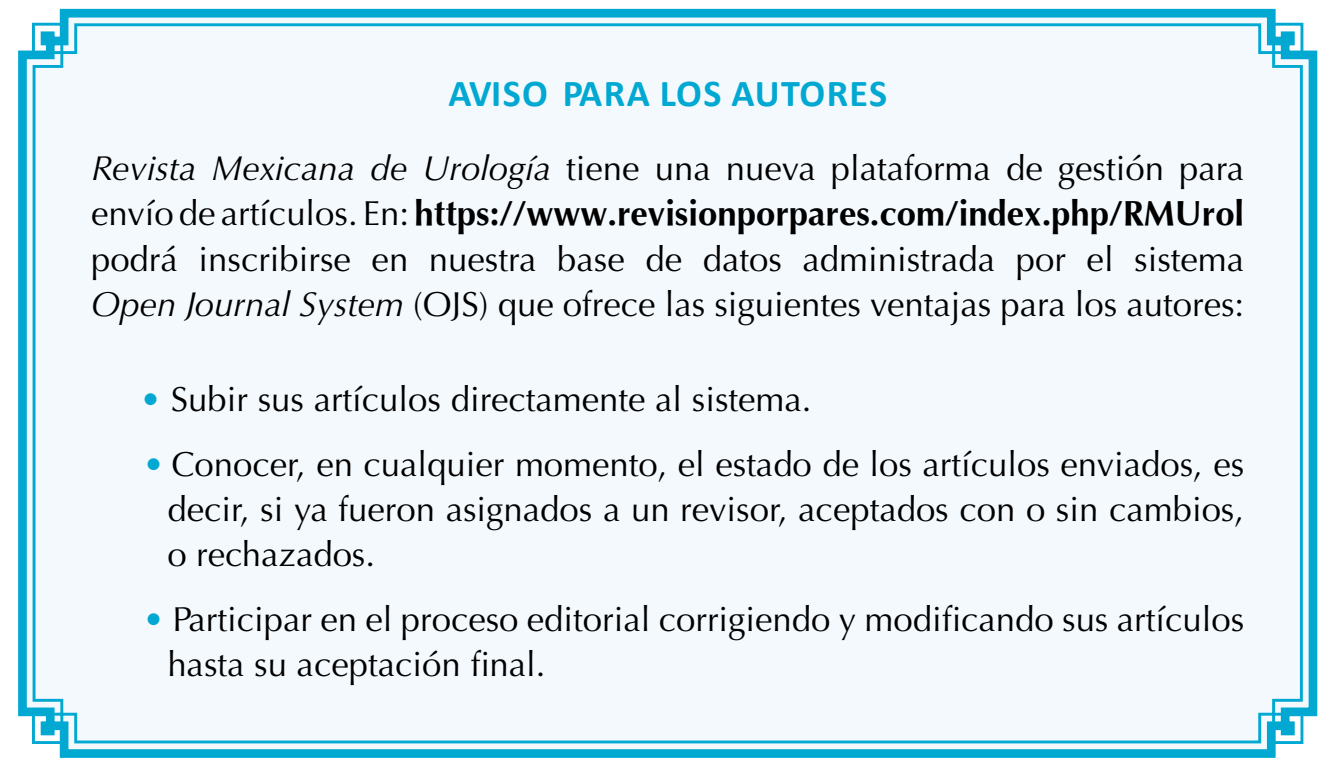

